\title{
K-Opioid Receptor Modulation of GABAergic Inputs onto Ventrolateral Periaqueductal Gray Dopamine Neurons
}

\author{
Chia Li ${ }^{a, b}$ Thomas L. Kash ${ }^{b, c}$ \\ ${ }^{a}$ Curriculum in Neurobiology, University of North Carolina Chapel Hill School of Medicine, Chapel Hill, NC, USA; \\ ${ }^{b}$ Bowles Center for Alcohol Studies, University of North Carolina Chapel Hill, Chapel Hill, NC, USA; ${ }^{C}$ Department of \\ Pharmacology, University of North Carolina Chapel Hill, Chapel Hill, NC, USA
}

\section{Keywords}

k-Opioid · Periaqueductal gray · Dopamine · Inhibition

\begin{abstract}
The k-opioid receptor (KOR) system has been implicated in the regulation of many behaviors including pain. While there are numerous studies suggesting KOR regulation of pain being mediated spinally, there have been reports of pain-like behaviors regulated by central KOR signaling. In particular, oxytocin-induced analgesia appears to be mediated by KOR receptors within the ventrolateral periaqueductal gray (vIPAG). We recently found that activation of dopamine (DA) neurons within the vIPAG is antinociceptive. In this study, we sought to determine the impact of KOR signaling on GABAergic inputs onto vIPAG DA neurons, and the mechanism through which KOR impacts these inputs. We found that activation of KOR reduced GABAergic transmission onto vIPAG DA neurons. In addition, our data suggest this effect is mediated presynaptically via the $G$ protein $\beta \gamma$-subunit. They raise the possibility that KOR activation disinhibits VIPAG DA neurons, which could lead to altered regulation of pain-related behaviors.

(c) 2019 S. Karger AG, Basel
\end{abstract}

\section{Introduction}

As a member of the opioid receptor family, $\kappa$-opioid receptors (KORs) have been shown to contribute considerably to analgesia, mood, and substance use disorder [14]. They are ubiquitously expressed in the brain, spinal cord, and periphery [5], and are activated endogenously by the opioid peptide dynorphin [6]. Canonically, KOR activation involves the inhibition of cyclic AMP production [7], which is mediated through coupling of the inhibitory $G$ protein $G_{a i / o}[8]$. Through dissociation of the a-subunit, which in turn recruits $\beta$-arrestin, KORs activate downstream mitogen-activated protein (MAP) kinases that affect transcription factor expression, such as ERK1/2 [9] and p38 [10]. The $\beta \gamma$-subunit can directly bind and inhibit calcium channels, as well as increase potassium channel conductance [11]. These direct effects on ion channel conductance have been found in several

The current affiliation of C.L. is National Institute of Diabetes and Digestive and Kidney Diseases (NIDDK), National Institutes of Health $(\mathrm{NIH})$, Bethesda, MD, USA. A preprint version of this paper is stored on bioRxiv (DOI 10.1101/389536).

\section{KARGER}

(C) 2019 S. Karger AG, Basel

E-Mail karger@karger.com

www.karger.com/mnp
Thomas L. Kash

Department of Pharmacology, University of North Carolina Chapel Hill 5023 Thurston Bowles

Chapel Hill, NC 27599-7178 (USA)

E-Mail tkash@email.unc.edu 
brain regions, ranging from the hippocampus to the dorsal root ganglia [12]. In addition, phosphorylated KOR activates ERK1/2, as well as phosphoinositide $3\left(\mathrm{PI}_{3}\right)$ kinase and protein kinase A (PKA). Evidence suggests that ERK signaling mediates KOR activation-induced attenuation of inhibitory neurotransmission in the bed nucleus of the stria terminalis (BNST) [13]. Furthermore, studies have shown that p38 MAP kinase signaling can regulate KOR-mediated inhibition of glutamate transmission in the BNST, and is required for negative affective states, which can be blocked by KOR antagonists [14]. The divergence in signaling pathways that mediate the effects of KOR activation is meaningful, as it suggests that biased agonists could be designed to selectively target specific pathways to engender different effects.

The A10dc group dopamine (DA) neurons project from the ventrolateral periaqueductal gray (vlPAG) to the extended amygdala - the BNST and the central amygdala, areas known to regulate stress, anxiety, and pain-related behaviors [15-18]. Recent studies by our group have found that chemogenetic activation of vlPAG DA neurons can alter pain-related behaviors. Beyond pain, these neurons have been implicated in arousal [19] and social behavior [20]. Of note, antinociception induced by oxytocin can be blocked by KOR antagonism in the PAG [21]. Mechanistically, it has been shown that KOR activation can modulate DA neurons in the ventral tegmental area (VTA) through multiple mechanisms. The postsynaptic effects of KOR appear to be limited to VTA DA neurons projecting to the prefrontal cortex [22], while KOR regulation of GABAergic plasticity appears to be presynaptically mediated [23].

Although KORs have been shown to be distributed widely in the PAG [24], their specific modulation of DA neurons has yet to be probed due to the heterogeneity of the PAG. Together, the existing behavioral and electrophysiological findings lead us to hypothesize that KOR may serve a role in modulating GABAergic inputs onto the vlPAG DA neurons.

\section{Materials and Methods}

\section{Animals and Husbandry}

Male TH-eGFP mice on a Swiss Webster background (aged 5-9 weeks) were bred and used in accordance with an animal use protocol approved by the University of North Carolina Chapel Hill (Institutional Animal Care and Use Committee). The mice were group housed in our colony room under a 12:12-h light-dark cycle, with lights on at 7:00 a.m. daily. They were given access to rodent chow and water ad libitum. Mating pairs of mice were created by GENSAT and obtained from the Mutant Mouse Resource and Re- search Center in North Carolina. In the TH-eGFP mouse line, the genome was modified to contain multiple copies of a modified BAC in which an eGFP reporter gene was inserted immediately upstream of the coding sequence of the gene for tyrosine hydroxylase $(\mathrm{TH})$. The data presented here were obtained from the transgenic mice maintained in-house.

\section{Electrophysiological Brain Slice Preparation}

The mice were decapitated under isoflurane anesthesia and their brains were rapidly removed and placed in ice-cold sucrose artificial cerebrospinal fluid (ACSF) (in mM) 194 sucrose, $20 \mathrm{NaCl}$, $4.4 \mathrm{KCl}, 2 \mathrm{CaCl}_{2}, 1 \mathrm{MgCl}_{2}, 1.2 \mathrm{NaH}_{2} \mathrm{PO}_{4}, 10.0$ glucose, and 26.0 $\mathrm{NaHCO}_{3}$ saturated with $95 \% \mathrm{O}_{2} / 5 \% \mathrm{CO}_{2}$. 300- $\mu$ m slices were prepared using a Leica VT1200 vibratome (Wetzlar, Germany).

\section{Slice Whole-Cell Electrophysiology}

Brain slices containing PAG were obtained and stored at approximately $30^{\circ} \mathrm{C}$ in a heated, oxygenated holding chamber containing ACSF (in mM) $124 \mathrm{NaCl}, 4.4 \mathrm{KCl}, 2 \mathrm{CaCl}_{2}, 1.2 \mathrm{MgSO}_{4}, 1$ $\mathrm{NaH}_{2} \mathrm{PO}_{4}, 10.0$ glucose, and $26.0 \mathrm{NaHCO}_{3}$ before being transferred to a submerged recording chamber maintained at approximately $30^{\circ} \mathrm{C}$ (Warner Instruments, Hamden, CT, USA). Recording electrodes (3-5 M $\Omega$ ) were pulled with a Flaming-Brown Micropipette Puller (Sutter Instruments, Novato, CA, USA), using thin-walled borosilicate glass capillaries. During inhibitory transmission experiments, recording electrodes were filled with (in $\mathrm{mM}) 70 \mathrm{KCl}, 65 \mathrm{~K}^{+}$-gluconate, $5 \mathrm{NaCl}, 10$ 4-(2-hydroxyethyl)-1-piperazineethanesulfonic acid, 2 QX-314, 0.6 EGTA, 4 ATP, and 0.4 GTP (pH 7.4, 290-295 mOsmol).

In experiments where postsynaptic GPCR signaling was blocked, GDP $\beta S$ was used to replace GTP in the internal solution. All experiments were conducted under the voltage clamp configuration, cells were held at $-70 \mathrm{mV}$, and inhibitory postsynaptic currents (IPSCs) were pharmacologically isolated with $3 \mathrm{~mm}$ kynurenic acid, to block AMPA ( $a$-amino-3-hydroxy-5-methyl-4isoxazole-propionic acid) and NMDA ( $N$-methyl-D-aspartate) receptor-dependent postsynaptic currents. To isolate miniature IPSCs (mIPSCs), tetrodotoxin $(0.5 \mu \mathrm{M})$ was added to the perfusing ACSF solutions described above. Signals were acquired via a MultiClamp 700B amplifier (Molecular Devices, Sunnyvale, CA, USA), digitized at $20 \mathrm{kHz}$, filtered at $3 \mathrm{kHz}$, and analyzed using Clampfit

Fig. 1. The $\kappa$-opioid receptor (KOR) agonist dynorphin A (dyn A; $300 \mathrm{nM}$ ) decreases miniature inhibitory postsynaptic currents (mIPSCs) in ventrolateral periaqueductal gray dopamine neurons via KOR actions. a Representative mIPSC traces at baseline (control; i) and after 10 min of 300-nM dynorphin A wash-on (ii). b Dynorphin A significantly decreased mIPSC frequency. c Dynorphin A had no effect on mIPSC amplitude. $\mathbf{d}$ The cumulative frequency of the MIPSC was shifted towards longer interevent intervals by dynorphin A. e The cumulative probability of the mIPSC amplitude was not affected by dynorphin A. f Dynorphin A no longer decreased mIPSC frequency in the presence of norbinaltorphimine (nor-BNI; $100 \mathrm{nM}$ ). g Dynorphin A had no effect on mIPSC amplitude in the presence of nor-BNI. $\mathbf{h}$ Nor-BNI alone had no effect on spontaneous (s)IPSC frequency. i Nor-BNI alone had no effect on sIPSC amplitude. ${ }^{*} p<0.05$.

(For figure see next page.) 


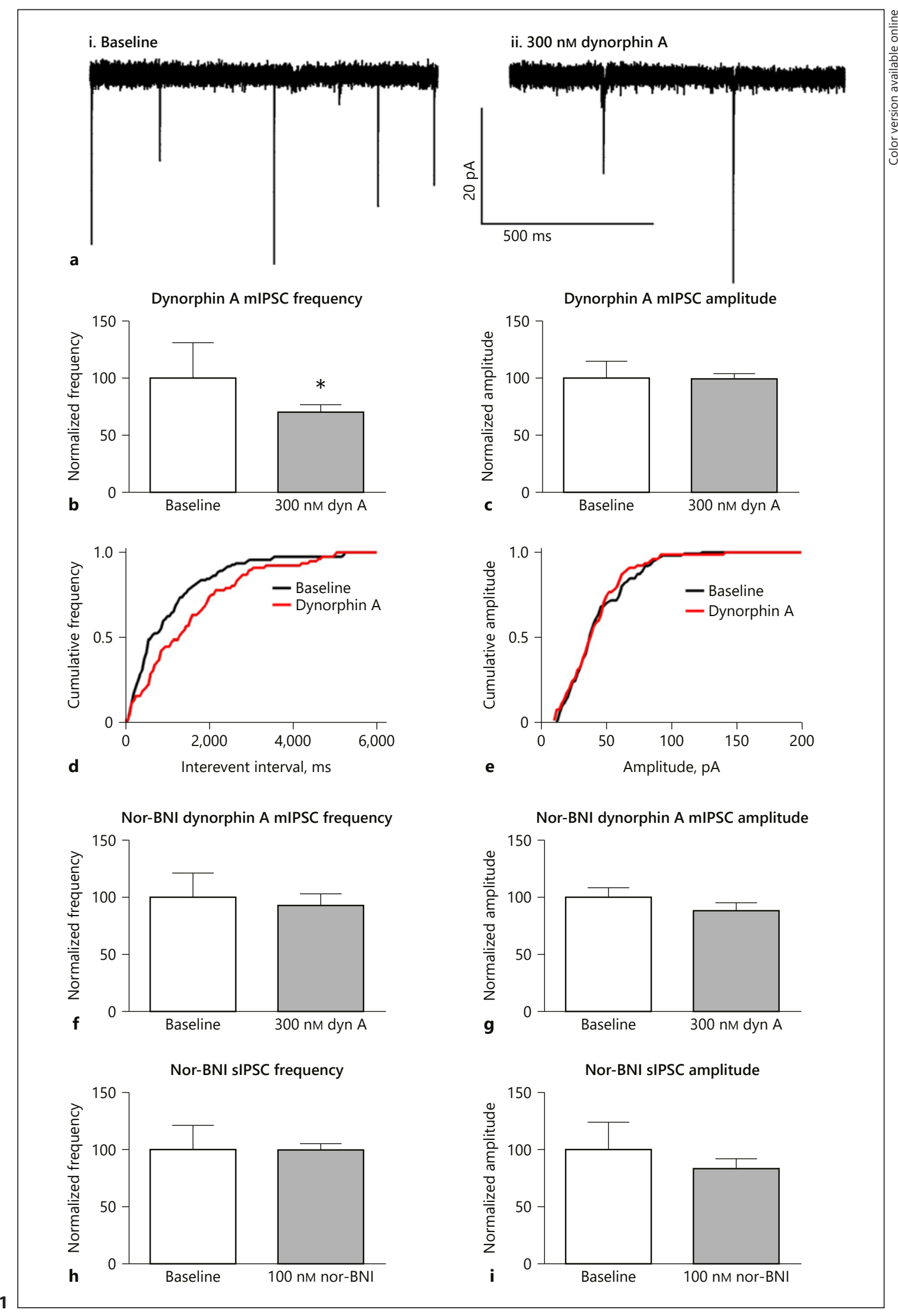




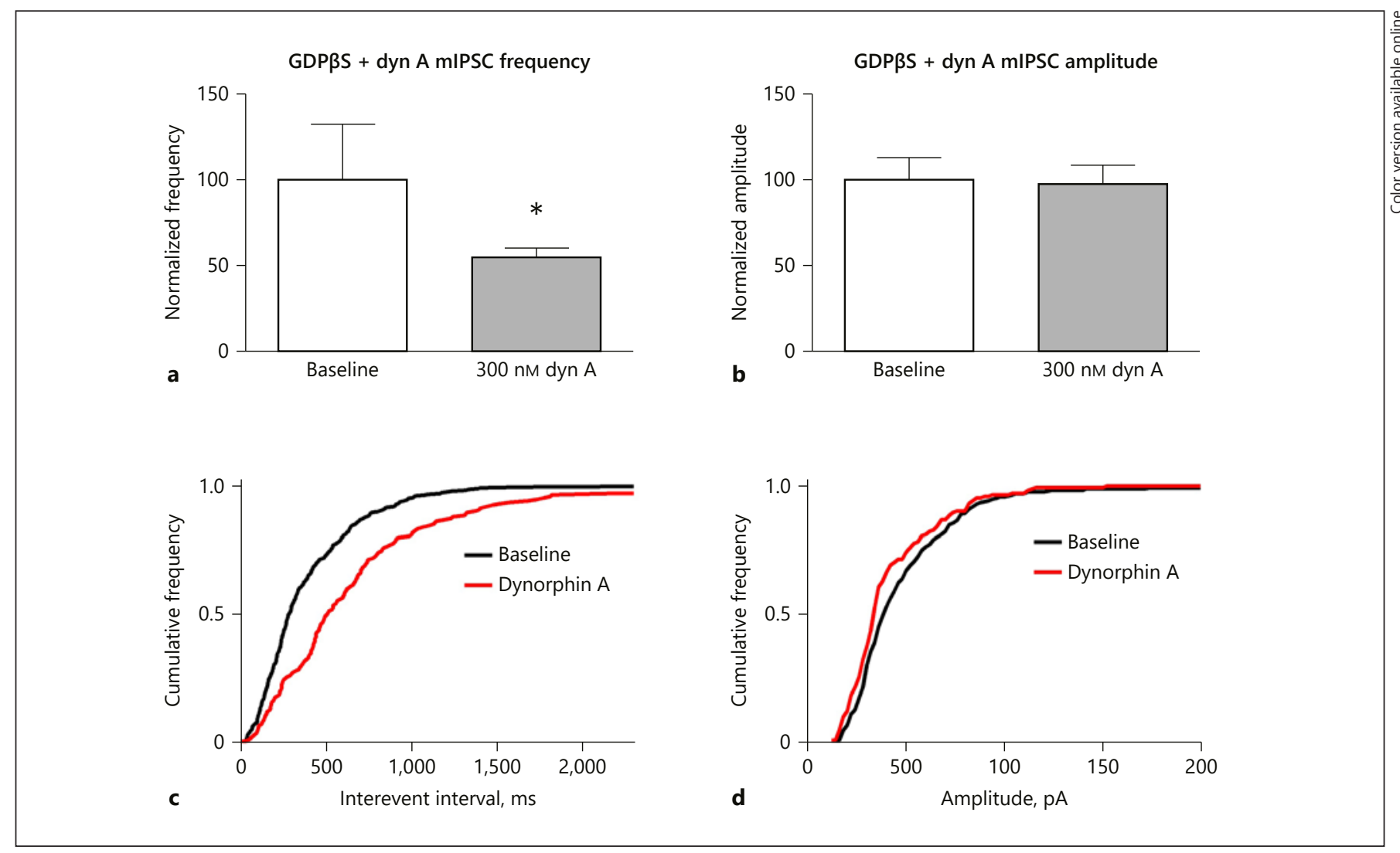

Fig. 2. The postsynaptic GPCR inhibitor GDP $\beta S$ (4 mM), diffused into the recorded ventrolateral periaqueductal gray dopamine neurons, did not block dynorphin A (dyn A) from attenuating miniature inhibitory postsynaptic current (mIPSC) frequency, suggesting a presynaptic mechanism. a Inhibition of postsynaptic GPCR did not hinder dynorphin A from attenuating mIPSC fre-

10.2 software (Molecular Devices). Input resistance and access resistance were continuously monitored during experiments. Experiments in which changes in access resistance were greater than $20 \%$ were not included in the data analysis.

\section{Statistics}

Effects of drugs during electrophysiological recordings were evaluated by comparing the magnitude of the dependent measure (mIPSC frequency and amplitude) between the baseline and washon (when the drug had reached its maximal effect at $10 \mathrm{~min}$ ) periods using paired $t$ tests. The effects of antagonists/blockers on the ability of the drugs to modulate synaptic transmission were compared using $t$ tests during the washout period. All values given for drug effects throughout the article are presented as mean \pm SEM.

\section{Drugs}

Dynorphin A (300 nM) and norbinaltorphimine (nor-BNI; 100 $\mathrm{nM}$ ) were purchased from Tocris (Ellisville, MO, USA) and dissolved in distilled water. BAPTA-AM $(50 \mu \mathrm{M})$, gallein $(100 \mu \mathrm{M})$, and wortmannin $(1 \mu \mathrm{M})$ were purchased from Tocris and dissolved in DMSO. 4-[4-(4-Fluorophenyl)-2-(4-[methylsulfinyl]phenyl)- quency. b Dynorphin A had no effect on mIPSC amplitude in the presence of GDP $\beta S$. c GDP $\beta$ S did not block dynorphin A's ability to shift the mIPSC cumulative frequency towards longer interevent intervals. d Dynorphin A had no effect on mIPSC amplitude cumulative probability. ${ }^{*} p<0.05$.

1H-imidazol-5-yl]pyridine (SB203580; $20 \mu \mathrm{M})$ and 4-aminopyridine (4-AP; $100 \mu \mathrm{M})$ were purchased from Ascent and dissolved in distilled water; $\alpha$-[amino([4-aminophenyl]thio)methylene]-2(trifluoromethyl)benzeneacetonitrile (SL327; $10 \mu \mathrm{M}$ ) was purchased from Ascent and dissolved in DMSO. Tetrodotoxin citrate $(500 \mathrm{nM})$ and kynurenic acid $(3 \mathrm{mM})$ were purchased from Abcam and dissolved in water. GDP $\beta S(4 \mathrm{mM})$ and RP-adenosine $3^{\prime}, 5^{\prime}$-cyclic monophophorothioate triethylammonium salt hydrate (RPcamps; $10 \mu \mathrm{M}$ ) were purchased from Sigma-Aldrich and dissolved in water. EGTA $(100 \mu \mathrm{M})$ was obtained from Fisher Scientific and dissolved in $1 \mathrm{M} \mathrm{NaOH}$.

\section{Results}

\section{Dynorphin A Attenuates GABAergic Input onto} vlPAG DA Neurons via a Presynaptic Mechanism

We first examined the effects of KOR activation on GABA synaptic transmission via bath application of the 


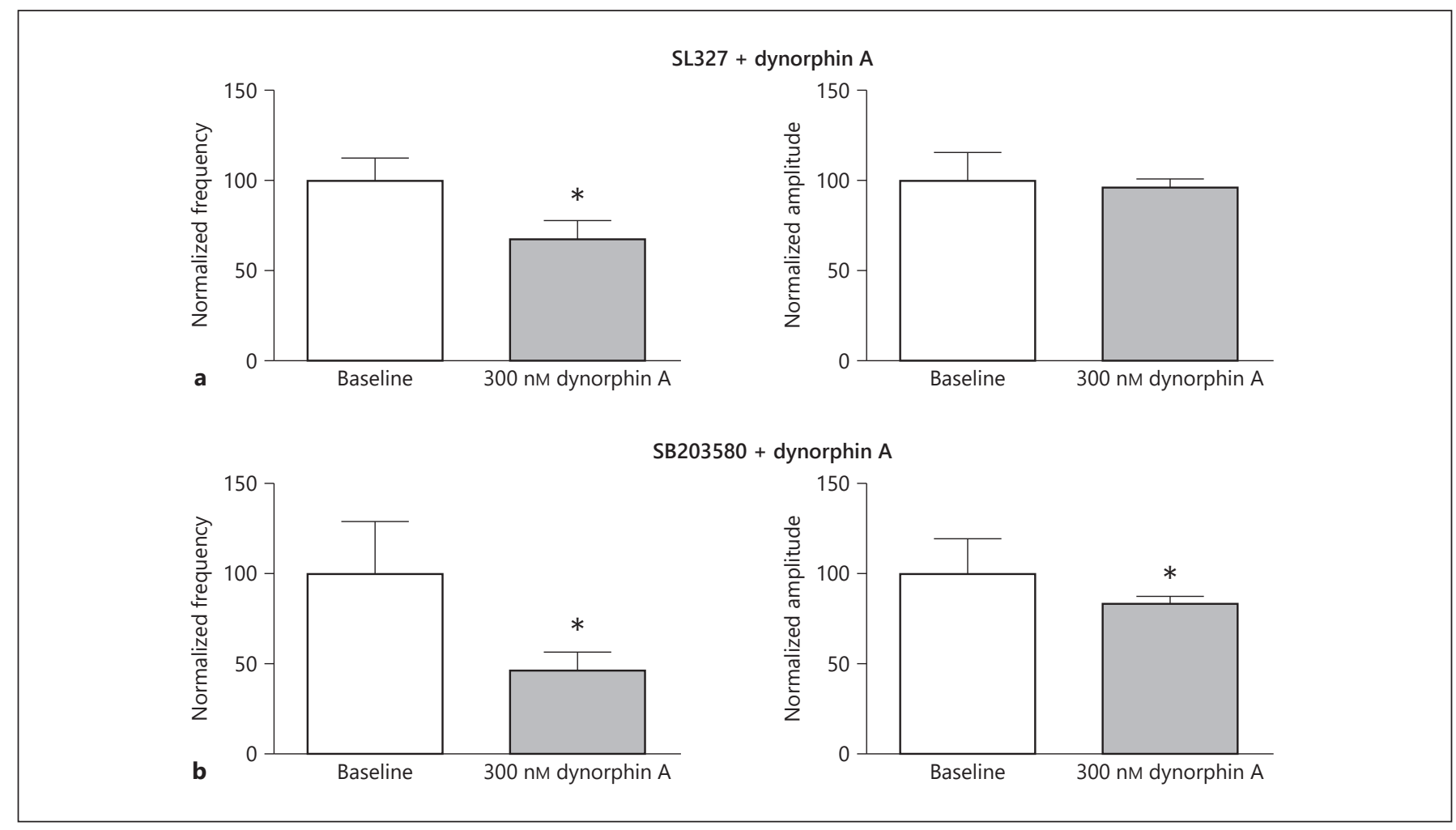

Fig. 3. The ERK1/2 inhibitor SL327 $(10 \mu \mathrm{M})$ and the p38 inhibitor SB203580 $(20 \mu \mathrm{M})$ did not hinder dynorphin A from attenuating miniature inhibitory postsynaptic current (mIPSC) frequency. a Dynorphin A significantly decreased mIPSC frequency in the presence of SL327. b Dynorphin A had no effect on mIPSC ampli-

endogenous ligand dynorphin A (300 nM). A 10-min bath application of dynorphin A significantly attenuated the mIPSC in vlPAG DA neurons (Fig. 1a; $n=5$ ). Specifically, a decrease was seen in mIPSC frequency $(69.5 \pm 7.4 \%$ of baseline, $p=0.03$; Fig. 1b, d), but not in amplitude $(99.3 \pm$ $4.8 \%$ of baseline; Fig. 1c, e), suggesting a presynaptic mechanism. To further confirm that the dynorphin effect observed was mediated through KOR activation, we incubated the slices in a selective KOR antagonist, nor-BNI (100 $\mathrm{nM}$ ), for $40 \mathrm{~min}$ before and during dynorphin wash-on $(n=5)$. In the presence of nor-BNI, dynorphin A application failed to produce effects on either mIPSC frequency ( $93.3 \pm 10.3 \%$ of baseline; Fig. 1 f) or amplitude $(89.4 \pm 6.1 \%$ of baseline; Fig. 1g). To assess the level of tonic KOR function, we investigated the effects of nor-BNI alone $(n=6)$ and found no effects on mIPSC frequency $(100.3 \pm 5.2 \%$ of baseline; Fig. $1 \mathrm{~h})$ and amplitude $(84.8 \pm 7.7 \%$ of baseline; Fig. 1i), indicating no tonic KOR activation in the vlPAG DA neurons. Combined, these data suggest a presynaptic effect of dynorphin on vlPAG DA neurons. tude in the presence of SL327. c Dynorphin A significantly decreased mIPSC frequency in the presence of SB203580. d Dynorphin A significantly decreased mIPSC amplitude in the presence of SB203580. * $p<0.05$.

We further verified this presynaptic mechanism via blockage of postsynaptic GPCR functions by the replacement of GTP with GDP $\beta$ S in the recording pipette, disrupting the exchange of GTP and GDP, thus interfering with downstream signaling cascades upon GPCR activation. With postsynaptic GPCR function impaired, the application of dynorphin A still decreased mIPSC frequency (54.7 \pm $5.8 \%$ of baseline, $n=6, p=0.04$; Fig. $2 \mathrm{a}, \mathrm{c}$ ), but not amplitude $(97.8 \pm 11.3 \%$ of baseline; Fig. $2 \mathrm{~b}, \mathrm{~d})$, providing additional support for dynorphin attenuating inhibitory input onto vlPAG DA neurons via a presynaptic mechanism.

\section{Effects of Dynorphin on GABA Are Not Mediated through MAP Kinase Signaling}

To identify the downstream signaling mechanisms through which KOR modulates GABAergic transmission, we examined the role of the MAP kinases ERK1/2 and $\mathrm{p} 38$. Brain slices were incubated in either a selective MEK inhibitor (SL327, $10 \mu \mathrm{M}, n=6$ ) or p38 inhibitor (SB203580, $20 \mu \mathrm{M}, n=5$ ) for $40 \mathrm{~min}$ before and during 


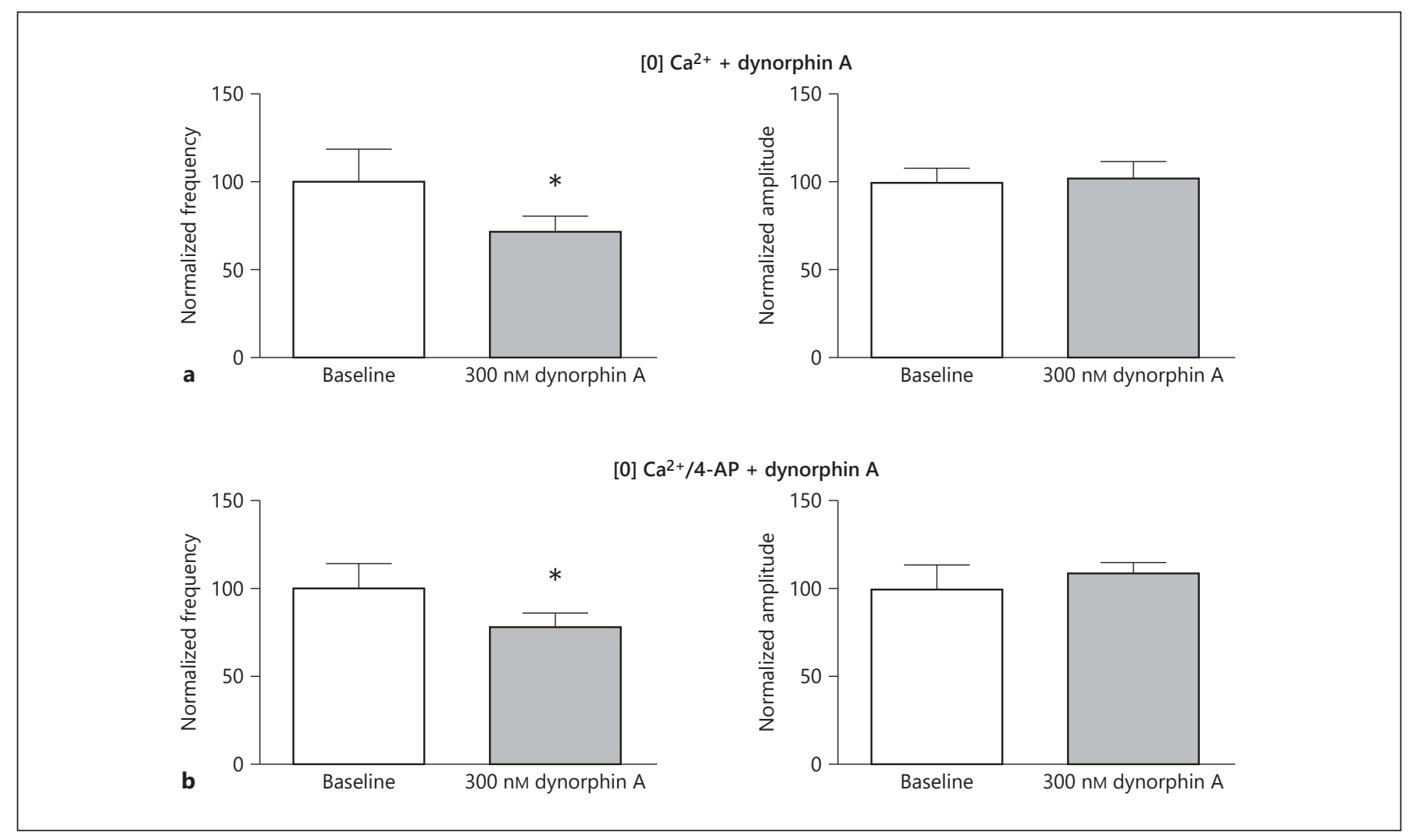

Fig. 4. $\kappa$-Opioid receptor (KOR) inhibition does not require calcium and potassium ion channels. The GPCR $\beta \gamma$-subunit inhibitor hindered dynorphin A from attenuating miniature inhibitory postsynaptic current frequency. a KOR/dynorphin A inhibition persisted even when all $\mathrm{Ca}^{2+}$ was removed by incubating slices in
$0 \mathrm{mM} \mathrm{Ca}^{2+} / 4 \mathrm{mM} \mathrm{Mg}^{2+}, 100 \mu \mathrm{M}$ EGTA with $50 \mu \mathrm{M}$ BAPTA-AM. b KOR/dynorphin A inhibition persisted even when all $\mathrm{Ca}^{2+}$ was removed and $\mathrm{K}^{+}$channels were blocked with 4 -aminopyridine (4-AP; $100 \mu \mathrm{M}) .{ }^{*} p<0.05$. dynorphin wash-on. In the presence of the MEK inhibitor SL327, dynorphin A significantly decreased mIPSC frequency (67.2 $\pm 10.6 \%$ of baseline, $p=0.04$; Fig. $3 a)$, but not amplitude (97.1 $\pm 4.3 \%$ of baseline; Fig. $3 \mathrm{a})$. In the presence of the p38 inhibitor SB203580, dynorphin A significantly decreased mIPSC frequency (46.6 \pm 9.9\% of baseline, $p<0.05$; Fig. 3b) and amplitude $(83.2 \pm 3.6 \%$ of baseline, $p=0.02$; Fig. $3 b)$. Neither SL327 nor p38 altered the dynorphin-induced attenuation of GABAergic input onto vlPAG DA neurons, suggesting this observation was not mediated through MAP kinase signaling.

\section{Effects of Dynorphin on GABA Are Not \\ Mediated through Calcium and Potassium Ion \\ Channel Conductance}

Agonist-induced dissociation of the $\beta \gamma$-subunit from the GPCR can directly influence the conductance of ion channels. Thus, we investigated the roles of calcium and potassium channels in dynorphin's modulation of the GABA-mediated IPSC. To test the role of calcium channels, we incubated the slices in calcium-free ACSF and the selective calcium chelators BAPTA-AM $(50 \mu \mathrm{M})$ and EGTA $(100 \mu \mathrm{M})$ for $1-2 \mathrm{~h}$ before recording, and continued to record from the slices in calcium-free ACSF in the presence of just EGTA, or EGTA plus 4-AP (100 $\mu \mathrm{M})$, to block potassium channels. In the calcium-free experiments, dynorphin A significantly decreased mIPSC frequency (71.9 $\pm 8.6 \%$ of baseline, $p=0.02, n=6$; Fig. $4 \mathrm{a})$, but not amplitude (102.3 $\pm 9.6 \%$ of baseline; Fig. $4 a)$. In the calcium-free experiments where potassium channels were blocked with 4-AP, dynorphin A still caused a significant decrease in mIPSC frequency $(77.8 \pm 8.0 \%$ of baseline, $p<0.05, n=8$; Fig. $4 \mathrm{~b}$ ), but not in amplitude (107.5 $\pm 5.7 \%$ of baseline; Fig. 4b). Taken together, these data suggest that KOR inhibition of GABA release in vlPAG DA neurons was not mediated via inhibition of calcium or potassium channels. 


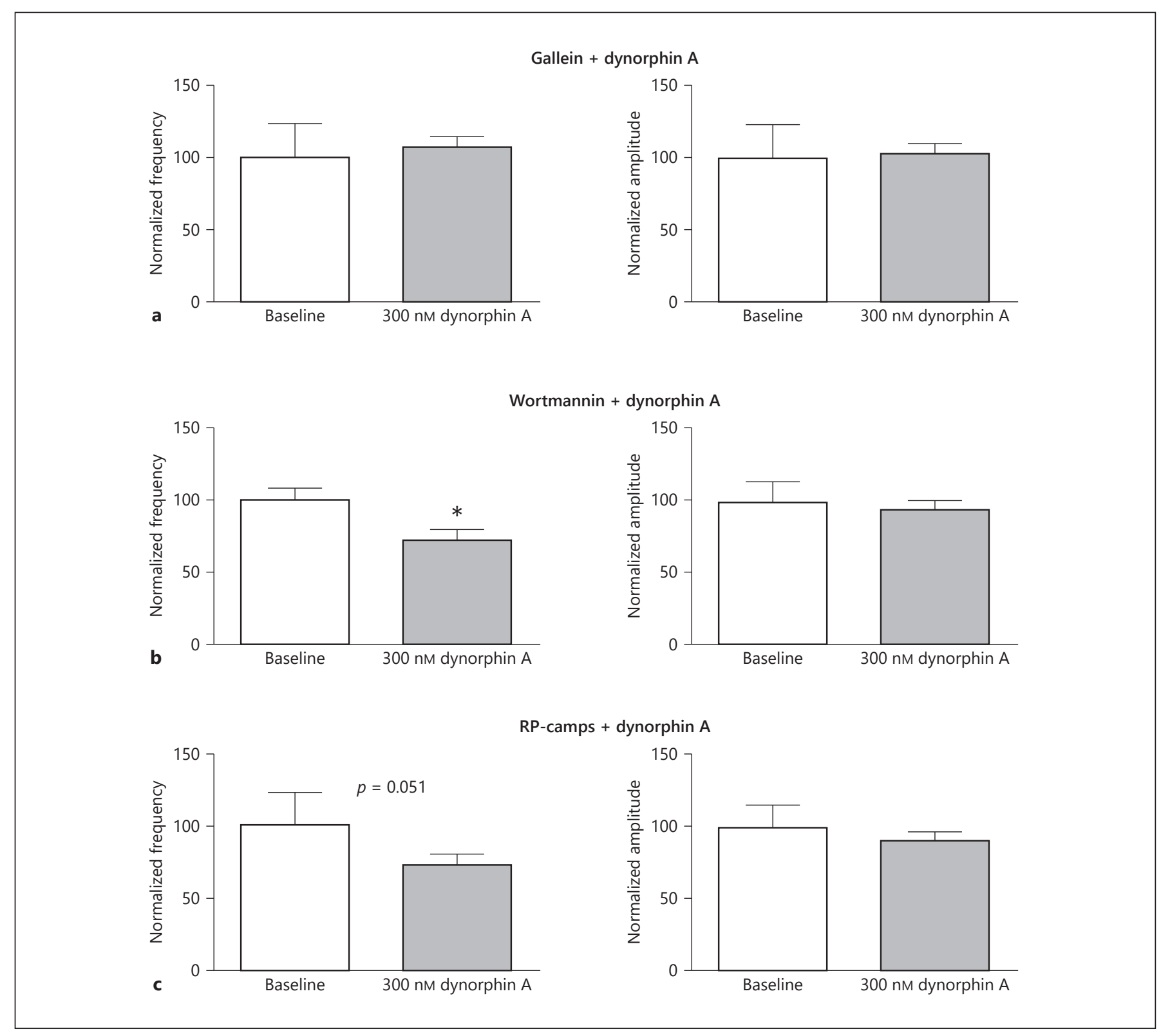

Fig. 5. Inhibition of $\mathrm{G}$ protein $\beta \gamma$-signaling but not phosphoinositide $3\left(\mathrm{PI}_{3}\right)$-kinase or protein kinase A (PKA) disrupts $\kappa$-opioid receptor effects on $\mathrm{GABA}$ release in the ventrolateral periaqueductal gray. a Gallein $(100 \mu \mathrm{M})$ prevented dynorphin A from inhibiting miniature inhibitory postsynaptic current (mIPSC) frequency. mIPSC amplitude remained unaffected. The $\mathrm{PI}_{3}$-kinase inhibitor

Effects of Dynorphin on GABA Are Mediated through $\beta \gamma$-Subunit-Dependent Signaling

We explored the role of the $\beta \gamma$-subunits in KOR inhibition of GABA transmission in vlPAG DA neurons beyond direct influence on ion channels by incubating slices in gallein $(100 \mu \mathrm{M})$, an inhibitor of $\mathrm{G}$ protein $\beta \gamma$ - wortmannin $(1 \mu \mathrm{M})$ and the PKA inhibitor RP-camps $(10 \mu \mathrm{M})$ did not hinder dynorphin A from attenuating mIPSC frequency. b Dynorphin A significantly decreased mIPSC frequency but had no effect on amplitude in the presence of wortmannin. c Dynorphin A decreased mIPSC frequency ( $p=0.051)$ but had no effect on amplitude in the presence of RP-camps.

subunit-dependent signaling. The previously observed KOR activation-induced reduction of GABA transmission was blocked in the presence of gallein $(n=6)$, with no significant changes in mIPSC frequency $(107.1 \pm 7.1 \%$ of baseline; Fig. $5 \mathrm{a})$ or amplitude $(102.8 \pm 7.4 \%$ of baseline; Fig. 5a). Together these data suggest that the effects 


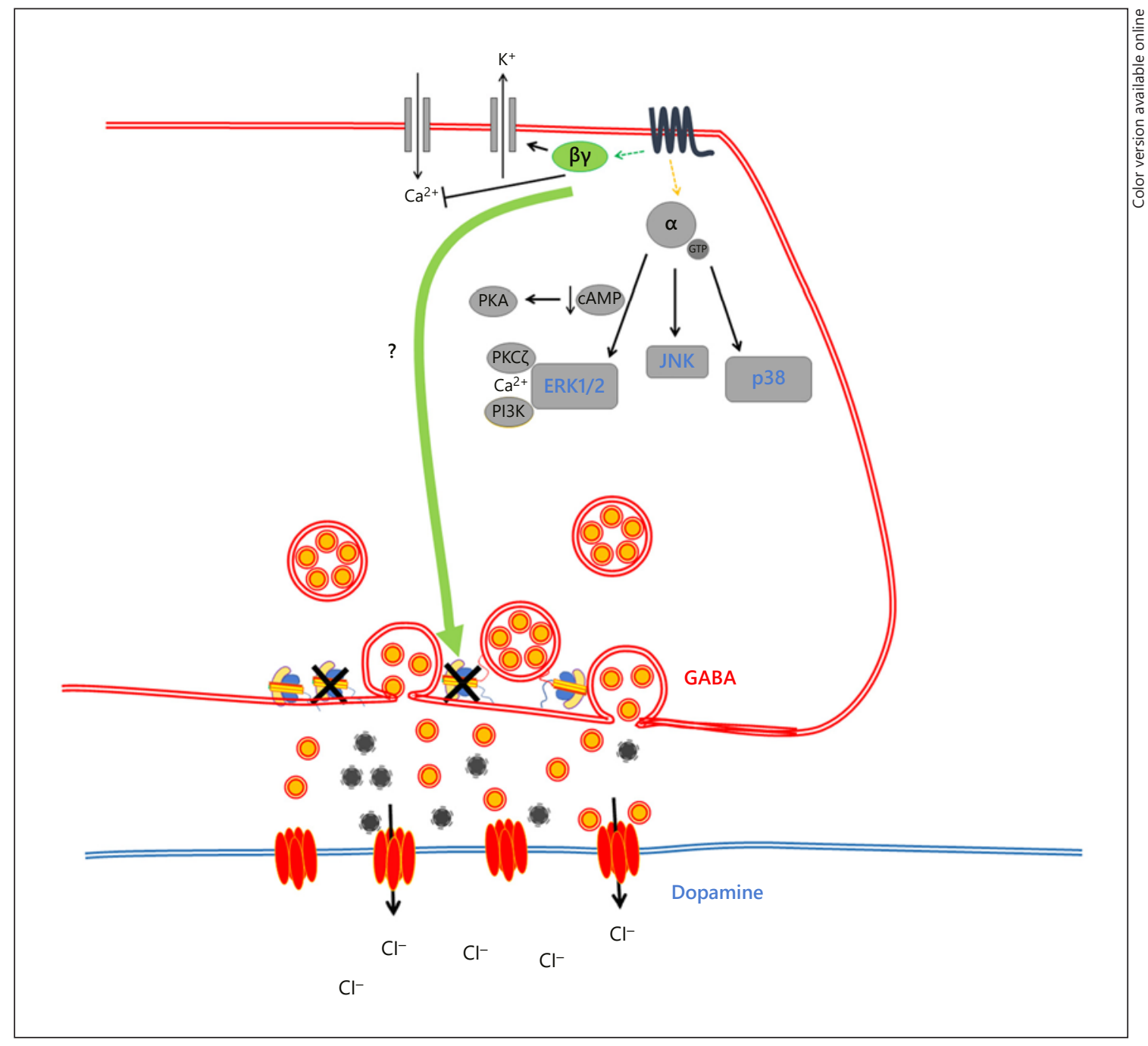

Fig. 6. $\kappa$-Opioid receptor regulates GABA release onto ventrolateral periaqueductal gray dopamine neurons via $\mathrm{G}$ protein $\beta \gamma$-signaling, potentially via regulation of the release machinery.

of KOR on GABAergic transmission were mediated via $\beta \gamma$-subunit signaling, but not through the change in conductance of ion channels.

Because gallein has been shown to not only inhibit the $\beta \gamma$-subunit but also activate $\mathrm{PI}_{3}$-kinase activity [25], we sought to clarify the role of $\mathrm{PI}_{3}$-kinase with wortmannin, a $\mathrm{PI}_{3}$-kinase inhibitor, as well as PKA signaling. We incubated slices in the $\mathrm{PI}_{3}$-kinase inhibitor wortmannin $(1 \mu \mathrm{M})$ or the PKA inhibitor RP-camps $(10 \mu \mathrm{M})$. Wortmannin did not block the previously observed decrease in GABA transmission frequency $(72.4 \pm 7.1 \%$ of baseline; Fig. $5 b)$. RP-camps also did not block the KOR-induced decrease in GABA frequency $(73.0 \pm 7.0 \%$ of baseline, $n=5, p=$ 0.051; Fig. 5c), and there were no effects on amplitude.
These data suggest that $\mathrm{PI}_{3}$-kinase and PKA do not mediate the KOR activation-induced decrease in GABA function in the vlPAG.

An overview of the mIPSC frequency raw values in vlPAG dopamine neurons under various treatments is shown in Table 1.

\section{Discussion}

vlPAG DA neurons have been implicated in a variety of behaviors, particularly the regulation of pain. Notably, KOR signaling in the vlPAG has been connected with oxytocin-induced analgesia. This study focused on deter- 
Table 1. Overview of mIPSC frequencies with various treatments

\begin{tabular}{lll}
\hline Drug & Treatment & $\begin{array}{l}\text { mIPSC frequency } \\
( \pm \text { SEM })\end{array}$ \\
\hline Dynorphin A & - & $1.4 \pm 0.4(n=5)$ \\
& $4-\mathrm{AP}$ & $1.1 \pm 0.4(n=7)$ \\
& {$[0] \mathrm{Ca}^{2+}$} & $0.4 \pm 0.1(n=6)$ \\
& {$[0] \mathrm{Ca}^{2+}+4-\mathrm{AP}$} & $0.6 \pm 0.1(n=8)$ \\
& GDP $\beta S$ & $1.3 \pm 0.4(n=6)$ \\
& Gallein & $1.6 \pm 0.4(n=6)$ \\
& Nor-BNI & $1.1 \pm 0.2(n=5)$ \\
& RP-camps & $1.4 \pm 0.3(n=5)$ \\
& SB203580 & $1.7 \pm 0.5(n=5)$ \\
& SL327 & $1.1 \pm 0.1(n=6)$ \\
& Wortmannin & $1.0 \pm 0.1(n=6)$ \\
\hline Nor-BNI & - & $1.0 \pm 0.2(\mathrm{sIPSC})(n=6)$ \\
\hline
\end{tabular}

mIPSC, miniature inhibitory postsynaptic current; sIPSC, spontaneous IPSC; 4-AP, 4-aminopyridine; nor-BNI, norbinaltorphimine.

mining the effects of KOR on inhibitory synaptic transmission onto vlPAG DA neurons - specifically, the downstream signaling mechanisms through which the effects take place. Briefly, we found that KOR can inhibit GABA release onto these neurons via a $G$ protein $\beta \gamma$-subunitdependent mechanism. The effect of KOR-induced inhibition of GABA release is consistent with findings in previous studies regarding the BNST [13], and it was abolished in the presence of a KOR antagonist (nor-BNI) in both regions, suggesting KOR-selective mediation. Although all vlPAG DA neurons recorded in this study showed a decrease in GABA-mediated IPSCs, studies on the VTA demonstrated that KOR attenuates inhibitory transmission selectively onto DA neurons that project to the basolateral amygdala [26, 27]. This raises the interesting possibility that KOR can serve to reduce the inhibitory drive onto DA neurons that project to amygdalar regions, perhaps supporting stress engagement of these pathways.

Using a pharmacological approach, we found that inhibition of $G$ protein $\beta \gamma$-subunit-dependent signaling successfully prevented the dynorphin A-induced decrease in mIPSC frequency. Because gallein has been shown to not only inhibit $\beta \gamma$-subunit but also activate $\mathrm{PI}_{3}$-kinase activity [25], we sought to clarify the role of $\mathrm{PI}_{3}$-kinase with wortmannin, a $\mathrm{PI}_{3}$-kinase inhibitor. Our results suggested that KOR-induced reduction of presynaptic inhibition lies outside of the actions of $\mathrm{PI}_{3}$-kinase. Further, our data demonstrated that in the vlPAG DA neurons, neither calcium nor potassium channels contributed to the presynaptic inhibition of GABA release. These results raised the possibility that dynorphin $\mathrm{A}$ could be activating KORs and directly affect the presynaptic release machinery in GABAergic inputs onto vlPAG DA neurons (Fig. 6). These data are similar to those from studies on KOR presynaptic inhibition of glutamatergic inputs in the hypothalamus, with effects persisting in the absence of presynaptic calcium channel activity and independent of cAMP signaling [28]. Although neither Iremonger and Bains [28] nor we were able to identify a precise molecular target through which dynorphin A inhibits presynaptic GABA release, the results are consistent with studies proposing a direct modulation of the exocytoticrelease machinery by the $\beta \gamma$-subunit of the $\mathrm{G}_{\mathrm{ai} / 0}$-coupled GPCR [29, 30].

It is currently unclear how KOR modulation of vlPAG DA neurons alters behavior. Given the prominent role that the vlPAG plays in pain and negative affect processing, as well as the correlation of KOR functions with emotional behaviors, it is tempting to speculate that KOR actions on this circuit are involved in these processes. Based on the hypothesis that KOR presynaptically inhibits GABAergic inputs, disinhibiting the vlPAG DA neurons to potentially modulate projection areas and related behaviors, further elucidation is needed regarding how KOR modulates the glutamatergic inputs, as well as the overall effect on activity of the vlPAG DA neurons. Future studies utilizing optogenetics to probe pathway-defined plasticity, as well as applying designer receptors exclusively activated by designer drugs (DREADD) to characterize pathway- and cell type-specific modulation, will likely shed light on this exciting possibility.

\section{Acknowledgement}

We thank the members of the Kash Lab for support throughout this project.

\section{Statement of Ethics}

Animals were used and experiments were conducted in accordance with an animal use protocol approved by the University of North Carolina Chapel Hill (Institutional Animal Care and Use Committee).

\section{Disclosure Statement}

The authors have no conflicts of interest to declare. 


\section{Funding Sources}

This project was funded by the Bowles Center for Alcohol Studies (University of North Carolina Chapel Hill; R00AA017668 [T.L.K.], R01AA019454 [T.L.K.], and P60AA011605 [T.L.K.]).

\section{Author Contributions}

Both authors conceived the studies; C.L. conducted the studies with assistance from T.L.K; C.L. wrote the initial manuscript; T.L.K. edited the manuscript; C.L. prepared the final manuscript.

\section{References}

1 Miller JM, Zanderigo F, Purushothaman PD, DeLorenzo C, Rubin-Falcone H, Ogden RT, et al. Kappa opioid receptor binding in major depression: a pilot study. Synapse. 2018 Sep; 72(9):e22042.

2 Tejeda HA, Shippenberg TS, Henriksson R. The dynorphin/א-opioid receptor system and its role in psychiatric disorders. Cell Mol Life Sci. 2012 Mar;69(6):857-96.

3 Koob GF. The dark side of emotion: the addiction perspective. Eur J Pharmacol. 2015 Apr;753:73-87.

4 Millan MJ. Kappa-opioid receptors and analgesia. Trends Pharmacol Sci. 1990 Feb;11(2): 70-6.

5 Peng J, Sarkar S, Chang SL. Opioid receptor expression in human brain and peripheral tissues using absolute quantitative real-time RT-PCR. Drug Alcohol Depend. 2012 Aug; 124(3):223-8.

6 Chavkin C, James IF, Goldstein A. Dynorphin is a specific endogenous ligand of the kappa opioid receptor. Science. 1982 Jan;215(4531): 413-5.

7 Minneman KP, Iversen IL. Enkephalin and opiate narcotics increase cyclic GMP accumulation in slices of rat neostriatum. Nature. 1976 Jul;262(5566):313-4.

8 Taussig R, Iñiguez-Lluhi JA, Gilman AG. Inhibition of adenylyl cyclase by Gi alpha. Science. 1993 Jul;261(5118):218-21.

9 Schattauer SS, Miyatake M, Shankar H, Zietz C, Levin JR, Liu-Chen LY, et al. Ligand directed signaling differences between rodent and human $\mathrm{k}$-opioid receptors. J Biol Chem. 2012 Dec;287(50):41595-607.

10 Bruchas MR, Macey TA, Lowe JD, Chavkin C. Kappa opioid receptor activation of p38 MAPK is GRK3- and arrestin-dependent in neurons and astrocytes. J Biol Chem. 2006 Jun;281(26):18081-9.

11 Dhawan BN, Cesselin F, Raghubir R, Reisine T, Bradley PB, Portoghese PS, et al. International Union of Pharmacology. XII. Classification of opioid receptors. Pharmacol Rev. 1996 Dec;48(4):567-92.
12 Grudt TJ, Williams JT. Opioid receptors and the regulation of ion conductances. Rev Neurosci. 1995 Jul-Sep;6(3):279-86.

13 Li C, Pleil KE, Stamatakis AM, Busan S, Vong L, Lowell BB, et al. Presynaptic inhibition of gamma-aminobutyric acid release in the bed nucleus of the stria terminalis by kappa opioid receptor signaling. Biol Psychiatry. 2012 Apr; 71(8):725-32.

14 Bruchas MR, Land BB, Aita M, Xu M, Barot SK, Li S, et al. Stress-induced p38 mitogenactivated protein kinase activation mediates kappa-opioid-dependent dysphoria. J Neurosci. 2007 Oct;27(43):11614-23.

15 Daniel SE, Rainnie DG. Stress modulation of opposing circuits in the bed nucleus of the stria terminalis. Neuropsychopharmacology. 2016 Jan;41(1):103-25.

16 Ahrens S, Wu MV, Furlan A, Hwang GR, Paik $\mathrm{R}, \mathrm{Li} \mathrm{H}$, et al. A central extended amygdala circuit that modulates anxiety. J Neurosci. 2018 Jun;38(24):5567-83.

17 Maruyama C, Deyama S, Nagano Y, Ide S, Kaneda K, Yoshioka M, et al. Suppressive effects of morphine injected into the ventral bed nucleus of the stria terminalis on the affective, but not sensory, component of pain in rats. Eur J Neurosci. 2018 Jan;47(1):40-7.

18 Tran L, Schulkin J, Greenwood-Van Meerveld B. Importance of CRF receptor-mediated mechanisms of the bed nucleus of the stria terminalis in the processing of anxiety and pain. Neuropsychopharmacology. 2014 Oct; 39(11):2633-45.

19 Cho JR, Treweek JB, Robinson JE, Xiao C, Bremner LR, Greenbaum A, et al. Dorsal raphe dopamine neurons modulate arousal and promote wakefulness by salient stimuli. Neuron. 2017 Jun;94(6):1205-1219.e8.

20 Matthews GA, Nieh EH, Vander Weele CM, Halbert SA, Pradhan RV, Yosafat AS, et al. Dorsal Raphe Dopamine Neurons Represent the Experience of Social Isolation. Cell. 2016 Feb;164(4):617-31.
21 Ge Y, Lundeberg T, Yu LC. Blockade effect of mu and kappa opioid antagonists on the antinociception induced by intra-periaqueductal grey injection of oxytocin in rats. Brain Res. 2002 Feb;927(2):204-7.

22 Margolis EB, Lock H, Chefer VI, Shippenberg TS, Hjelmstad GO, Fields HL. Kappa opioids selectively control dopaminergic neurons projecting to the prefrontal cortex. Proc Natl Acad Sci USA. 2006 Feb;103(8):2938-42.

23 Polter AM, Barcomb K, Chen RW, Dingess PM, Graziane NM, Brown TE, et al. Constitutive activation of kappa opioid receptors at ventral tegmental area inhibitory synapses following acute stress. eLife. 2017 Apr;6:6.

24 Gutstein HB, Mansour A, Watson SJ, Akil H, Fields HL. Mu and kappa opioid receptors in periaqueductal gray and rostral ventromedial medulla. Neuroreport. 1998 Jun 1;9(8):177781.

25 Ukhanov K, Brunert D, Corey EA, Ache BW. Phosphoinositide 3-kinase-dependent antagonism in mammalian olfactory receptor neurons. J Neurosci. 2011 Jan;31(1):273-80.

26 Ford CP, Mark GP, Williams JT. Properties and opioid inhibition of mesolimbic dopamine neurons vary according to target location. J Neurosci. 2006 Mar;26(10):2788-97.

27 Ford CP, Beckstead MJ, Williams JT. Kappa opioid inhibition of somatodendritic dopamine inhibitory postsynaptic currents. J Neurophysiol. 2007 Jan;97(1):883-91.

28 Iremonger KJ, Bains JS. Retrograde opioid signaling regulates glutamatergic transmission in the hypothalamus. J Neurosci. 2009 Jun;29(22):7349-58.

29 Blackmer T, Larsen EC, Takahashi M, Martin TF, Alford S, Hamm HE. G protein betagamma subunit-mediated presynaptic inhibition: regulation of exocytotic fusion downstream of Ca2+ entry. Science. 2001 Apr;292(5515): 293-7.

30 Delaney AJ, Crane JW, Sah P. Noradrenaline modulates transmission at a central synapse by a presynaptic mechanism. Neuron. 2007 Dec;56(5):880-92. 